\title{
Radiographic Changes of the Nasal Septal Body Among Patients With Sinonasal Diseases
}

\author{
January E. Gelera ${ }^{1,2} \cdot$ Devendra Ojar ${ }^{1} \cdot$ Jae Hyun Lim $^{1} \cdot$ Jee Hye Wee ${ }^{1} \cdot$ Jeong-Whun Kim ${ }^{1}$ Chae-Seo Rhee ${ }^{1,3,4}$ \\ ${ }^{I}$ Department of Otorhinolaryngology-Head and Neck Surgery, Seoul National University Bundang Hospital, Seoul National University College of \\ Medicine, Seoul, Korea; ' 2 Department of Otorhinolaryngology-Head and Neck Surgery, University of Santo Tomas Hospital, Manila, Philippines; \\ ${ }^{3}$ Research Center for Sensory Organs and Institute of Allergy and Clinical Immunology, Seoul National University Medical Research Center,
}

Seoul, Korea

Objectives. This study aims to determine the anatomical changes occurring in the nasal septal body (NSB) among patients with sinonasal disease and compares the measurements obtained from patients without sinonasal disease.

Methods. This was a retrospective study involving review of 405 (93 without and 212 with sinonasal disease) plain paranasal sinus computed tomography (PNS CT) on coronal view at a tertiary training hospital, which was done consecutively from January 2014 to December 2015. NSB measurements from 3 areas were done: anterior part (A), located anterior and superior to inferior turbinate; middle or widest $(\mathrm{M})$ part, located anterior to middle turbinate and superior to inferior turbinate and posterior $(\mathrm{P})$ part, located within the anterior $1 / 3$ of middle turbinate not going beyond the crista galli. Posterior part of septum (sP) was measured at the area of horizontal attachment of middle turbinate to the lateral nasal wall and superior turbinate to represent the less vasoactive part of the septum. Demographic data and NSB diameters were also analyzed.

Results. The mean NSB diameter measurements were significantly larger among the diseased group (disease vs. control; A: $6.88 \mathrm{~mm}$ vs. $5.92 \mathrm{~mm}, P=0.001$; M: $12.74 \mathrm{~mm}$ vs. $10.47 \mathrm{~mm}, P=0.001$; P: $8.35 \mathrm{~mm}$ vs. $6.79 \mathrm{~mm}, P=0.001$ ). A similar observation in $\mathrm{sP}$ part ( $3.35 \mathrm{~mm}$ vs. $2.54 \mathrm{~mm}, P=0.014)$ was noted. When grouped by age, among the control group, older subjects had considerable decrease in NSB size in all points of measurements except for sP $(P>0.05)$. Such reduction in size is noticeable for those in their 4th, 5th, 6th, and 7th decades of life. For the diseased group, a trend of increasing NSB and sP size was noted as the subjects are getting older. However, only the anterior part (A) of NSB reached statistical significance $(P=0.016)$.

Conclusion. With aging we expect nasal mucosal atrophy among normal subjects. However, patients with chronic sinonasal disease showed thickened nasal mucosa. Further study for the reversibility of thickened mucosa is needed.

Keywords. Nasal Septal Body; Nasal Septal Swell Body

\section{INTRODUCTION}

- Received January 16, 2017

Revised April 11, 2017

Accepted May 15, 2017

- Corresponding author: Chae-Seo Rhee

Department of Otorhinolaryngology-Head and Neck Surgery, Seoul

National University Bundang Hospital, 82 Gumi-ro 173beon-gil, Bundang-

gu, Seongnam 13620, Korea

Tel: +82-31-787-7401, Fax: +82-31-787-4057

E-mail: csrhee@snu.ac.kr
The nasal septal body (NSB) is a distinct anatomical structure of the anterior nasal septum, composed of thicker cartilage and mucosa as compared to the rest of the nasal septum. It is clinically seen as a soft-tissue thickening located superior to the inferior turbinate, and anterior to the middle turbinate [1-3]. This mucosal swelling is quickly noted on both nasal endoscopy and cross-sectional imaging. However, this focal midline prominence

Copyright $@ 2017$ by Korean Society of Otorhinolaryngology-Head and Neck Surgery.

This is an open-access article distributed under the terms of the Creative Commons Attribution Non-Commercial License (http://creativecommons.org/licenses/by-nc/4.0)

which permits unrestricted non-commercial use, distribution, and reproduction in any medium, provided the original work is properly cited. 
is either often ignored or confused for a high septal deviation.

In a literature search limited information is written about the NSB. The studies mainly focused on histology and gross anatomic morphology. The septal body has been described as a dynamic structure that can regulate nasal airflow and humidify inspired air. This is primarily due to the structure's vasoactive and glandular properties [2,4]. Furthermore, it has been emphasized that its proximity to the internal nasal valve (INV) can markedly alter nasal resistance and airflow because of its possible effect on the cross-sectional diameter of this region $[2,4,5]$.

In addition, the NSB shares similar histologic morphology with the inferior turbinate [2]. It can be presumed that the septal body can also undergo similar mucosal changes as seen in hypertrophy of the inferior turbinates in cases of chronic sinonasal diseases (e.g., allergic rhinitis [AR] and chronic rhinosinusitis [CRS]). The turbinate mucosal enlargement is mainly due to fibrosis as the reactive outcome of chronic inflammation. When fibrosis sets in, the increase in size is difficult to reverse and thus, conventional treatment such as nasal decongestants and corticosteroids may have lesser effects [6]. Similarly, NSB could develop hypertrophic change and interfere nasal airflow in chronic inflammation of the nasal cavity. Thus, we assume that the size of NSB could be changed in chronic inflammatory diseases of the nose.

The authors thought that NSB can be more prominent in patients with chronic sinonasal inflammatory disease like AR and CRS. Because even though both had differing pathophysiology where AR is an immunoglobulin-E mediated inflammatory disease upon exposure to allergen while CRS is a clinical syndrome characterized by persistent symptomatic inflammation of the nasal mucosa due to dysfunctional interplay between host and environment, the hallmark of these diseases is chronic inflammation. In addition, most radiographic papers about NSB only measured the widest diameter on cross-sectional coronal computed tomography $[1-4,7,8]$. These studies did not include the linear dimensions of the anterior and posterior parts of the fusiformshaped septal structure and the less vasoactive part of the septum. These measurements could show that these components could also undergo significant changes with the influence of chronic inflammation. Furthermore, the authors think that the

\section{H I}

- Radiographic changes (paranasal sinus computed tomography coronal view) of nasal septal body (NSB) were reviewed among 405 patients with and without sinonasal disease.

- Mean NSB diameter is significantly larger among the diseased group $(P=0.01)$.

- As the individual gets older, NSB diameter was observed to significantly decrease in size $(P=0.01)$.

- The opposite was observed among the diseased group however the change is not statistically significant $(P>0.05)$. overdevelopment of the anterior and widest section of NSB have more anatomic relevance in altering the parabolic flow of inspired air. Thus, this study aims to determine the radiographic changes of the NSB among patients with chronic sinonasal inflammatory disease.

\section{MATERIALS AND METHODS}

This was a retrospective study conducted at a tertiary training hospital involving a review of paranasal sinus computed tomography (PNS CT) scan among patients with and without PNS disease. The CT review was done in a consecutive manner from January 2014 to December 2015. The Institutional Review Board of Seoul National University Bundang Hospital permitted this study (No. B-1611-371-101).

\section{Study population}

The study included 405 PNS CT scans. Patients diagnosed with $\mathrm{AR}$ and chronic rhinosinusitis with (CRSwNP) or without nasal polyposis (CRSsNP) would be classified under the diseased group. While patients wherein PNS CT scan were requested for surgical access for brain tumors like craniopharyngioma, meningioma, and pituitary adenoma or for malocclusion, dental problem and facial pain, without any clinical and radiological findings related to PNS disease served as the control group. Both groups were further categorized into age groups, in years: $\leq 19$, 20-29, 30-39, 40-49, 50-59, and $>60$.

Three hundred twelve subjects were identified with sinonasal diseases (203 AR, 50 AR and CRSsNP, 32 AR and CRSwNP, 16 CRSsNP, and 11 CRSwNP) while 93 had no sinonasal diseases. The control group consisted of 46 males $(49.5 \%)$ and 47 females $(50.5 \%)$ with mean age of $43.38 \pm 18.13$ years old. The diseased group consisted of 212 males $(67.9 \%)$ and 100 females $(32.1 \%)$ with mean age of $35.13 \pm 16.22$ years old. The average age difference between the 2 groups was statistically significant.

The AR diagnosis was made using the Allergic Rhinitis and its Impact on Asthma guideline and with confirmatory skin pricks test (SPT) and multiallergen screening test (MAST) [9]. The SPT is considered positive if the wheal diameter is $3 \mathrm{~mm}$ greater than the negative (saline) control and MAST result of as low as 27-65 LU/mL was interpreted as positive [10]. CRS diagnosis on the other hand were made using The American Academy of Otolaryngology-Head and Neck Surgery Adult Sinusitis guidelines and Lund-Mackay score of greater than 4 [11,12].

This study excluded PNS CT scans from patients whose septum cannot be delineated due to massive polyposis and severe septal deviation; and those who underwent nasal surgery.

\section{PNS CT evaluation}

As shown in Fig. 1, NSB was identified, and the horizontal diameter was measured. Additional measurement posterior to the 


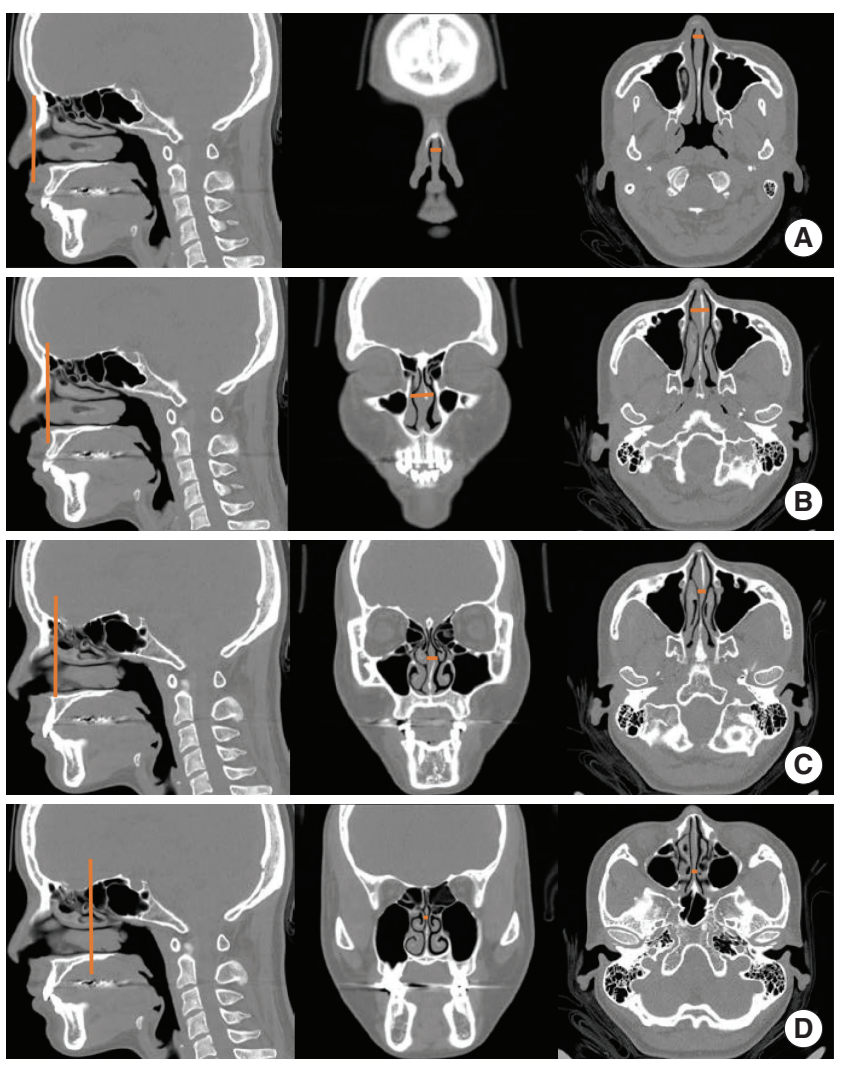

Fig. 1. Measurement of nasal septal body (NSB) and posterior sep tum. The red lines in left side of figures indicate the level of measurement, and those in middle and right sides of figures indicate the actual measurement. (A) NSB anterior part, (B) NSB middle or widest part, (C) NSB posterior part, and (D) posterior part of septum.

NSB structure was done to represent the less vasoactive area of the nasal septum. NSB was divided into 3 segments as follows: anterior part (A), located anterior and superior to inferior turbinate; middle or widest part $(\mathrm{M})$, located anterior to middle turbinate and superior to inferior turbinate and posterior part $(\mathrm{P})$, located within the anterior $1 / 3$ of middle turbinate not going beyond the crista galli. Posterior part of the septum (sP) was measured at the area of horizontal attachment of middle turbinate to the lateral nasal wall and superior turbinate. The first author (JEG) performed all measurements.

Data analysis was performed using IBM SPSS ver. 22 (IBM Co., Armonk, NY, USA). The independent $t$-test and ANOVA were used for statistical analysis of variables. The $P$-values of $<0.05$ were considered significant.

\section{RESULTS}

The horizontal diameter of NSB and posterior septum were tabulated in Table 1. The mean NSB diameter measurements were significantly larger among the diseased group (disease vs. control;
Table 1. Nasal septal body diameter $(\mathrm{mm})$ between diseased and control groups from 4 points of the nasal septum

\begin{tabular}{lccc}
\hline Point of measurement & $\begin{array}{c}\text { Diseased group } \\
(n=312)\end{array}$ & $\begin{array}{c}\text { Control group } \\
(n=93)\end{array}$ & $P$-value \\
\hline Anterior part & $6.88 \pm 0.96$ & $5.92 \pm 0.84$ & 0.001 \\
Middle/widest part & $12.74 \pm 1.62$ & $10.47 \pm 1.32$ & 0.001 \\
Posterior part & $8.35 \pm 1.54$ & $6.79 \pm 1.36$ & 0.001 \\
Posterior part of septum & $3.35 \pm 5.65$ & $2.54 \pm 0.69$ & 0.014 \\
\hline
\end{tabular}

Values are presented as mean \pm standard deviation.

A: $6.88 \pm 0.96 \mathrm{~mm}$ vs. $5.92 \pm 0.84 \mathrm{~mm}, P=0.001 ; \mathrm{M}: 12.74 \pm 1.62$ $\mathrm{mm}$ vs. $10.47 \pm 1.32 \mathrm{~mm}, P=0.001 ; \mathrm{P}: 8.35 \pm 1.54 \mathrm{~mm}$ vs. $6.79 \pm$ $1.36 \mathrm{~mm}, P=0.001)$. A similar observation in sP part ( $3.35 \pm 5.65$ $\mathrm{mm}$ vs. $2.54 \pm 0.69 \mathrm{~mm}, P=0.014$ ) was noted. When grouped by age, among the control group, older subjects had narrower NSB diameter in all points except for $\mathrm{sP}(P>0.05)$ (Table 2$)$. Such reduction in size was noticeable for those in their 4th, 5th, 6th, and 7th decades of life. For the diseased group, a trend of increasing NSB and SP size was noted as the subjects advance in age. However, only the anterior part (A) of NSB reached statistical significance $(P=0.016)$.

\section{DISCUSSION}

The role of NSB in nasal airflow has always been poorly understood. Various terminologies such as nasal septal swell body, anterior septal tubercle, septal turbinate, intumescentia nasi anterior, and Kisselbach's body were used to describe this prominent structure of the anterior nasal chamber in the literature [2]. These seemed to characterize septal body's function. However, evidence of its influence in nasal airflow is yet to be determined. To date, there is no nasal aerodynamic study relevant to the septal swell body or an in vivo study measuring the changes occurring in healthy, diseased, and decongested state. These investigations are valuable to validate the hypothesis that NSB can indeed alter nasal airflow and resistance. The similarities in the histological and functional properties of NSB and inferior turbinate are as follows: both possess venous sinusoids and glandular properties although NSB shows a much lesser degree and both can change in size and shape in response to nasal cycle. Thus, this led the authors to think NSB may play a role in nasal aerodynamics [2].

The INV, an important anatomical landmark in the nose, is the area where the inspiratory and expiratory air currents get turbulent. Any further narrowing of this region can significantly affect the nasal airflow hence can lead to subjective nasal obstruction $[1,4,5,13]$. The authors of the current study, as well as some experts, think that NSB should be considered as part of INV, along with the caudal inferior turbinate, septum, and upper lateral cartilage $[5,13]$. Thus, attention should be given to NSB when presented with restricted nasal airway and when doing surgical planning to correct narrowed nasal valve area. 
Table 2. Nasal septal body diameter by age group among control and diseased group

\begin{tabular}{|c|c|c|c|c|c|c|c|}
\hline \multirow{2}{*}{ Point of measurement } & \multirow{2}{*}{ Age (yr) } & \multicolumn{3}{|c|}{ Control group $(n=93)$} & \multicolumn{3}{|c|}{ Diseased group $(n=312)$} \\
\hline & & No. & Diameter (mm) & $P$-value & No. & Diameter (mm) & $P$-value \\
\hline \multirow[t]{7}{*}{ Anterior part } & & & & 0.001 & & & 0.016 \\
\hline & $\leq 19$ & 12 & $6.31 \pm 0.73$ & & 59 & $6.48 \pm 0.74$ & \\
\hline & $20-29$ & 13 & $6.69 \pm 1.21$ & & 86 & $6.90 \pm 1.10$ & \\
\hline & 30-39 & 14 & $6.07 \pm 0.66$ & & 47 & $6.90 \pm 0.73$ & \\
\hline & $40-49$ & 17 & $5.94 \pm 0.58$ & & 41 & $7.02 \pm 0.86$ & \\
\hline & $50-59$ & 13 & $5.65 \pm 0.53$ & & 50 & $7.09 \pm 1.10$ & \\
\hline & $\geq 60$ & 24 & $5.36 \pm 0.84$ & & 29 & $6.98 \pm 1.00$ & \\
\hline \multirow[t]{7}{*}{ Middle/widest part } & & & & 0.001 & & & 0.733 \\
\hline & $\leq 19$ & 12 & $11.66 \pm 1.05$ & & 59 & $12.46 \pm 1.53$ & \\
\hline & $20-29$ & 13 & $11.27 \pm 0.85$ & & 86 & $12.73 \pm 1.62$ & \\
\hline & $30-39$ & 14 & $10.99 \pm 1.20$ & & 47 & $12.80 \pm 1.80$ & \\
\hline & $40-49$ & 17 & $10.43 \pm 0.95$ & & 41 & $12.84 \pm 1.50$ & \\
\hline & $50-59$ & 13 & $9.71 \pm 0.99$ & & 50 & $12.94 \pm 1.71$ & \\
\hline & $\geq 60$ & 24 & $9.57 \pm 1.29$ & & 29 & $12.84 \pm 1.65$ & \\
\hline \multirow[t]{7}{*}{ Posterior part } & & & & 0.001 & & & 0.427 \\
\hline & $\leq 19$ & 12 & $8.43 \pm 1.32$ & & 59 & $8.10 \pm 1.47$ & \\
\hline & $20-29$ & 13 & $7.41 \pm 1.44$ & & 86 & $8.55 \pm 1.56$ & \\
\hline & $30-39$ & 14 & $7.14 \pm 0.87$ & & 47 & $8.07 \pm 1.19$ & \\
\hline & $40-49$ & 17 & $6.86 \pm 0.94$ & & 41 & $8.46 \pm 1.64$ & \\
\hline & $50-59$ & 13 & $5.99 \pm 1.22$ & & 50 & $8.38 \pm 1.79$ & \\
\hline & $\geq 60$ & 24 & $5.82 \pm 0.77$ & & 29 & $8.47 \pm 1.58$ & \\
\hline \multirow[t]{7}{*}{ Posterior part of septum } & & & & 0.099 & & & 0.815 \\
\hline & $\leq 19$ & 12 & $3.02 \pm 0.47$ & & 59 & $3.03 \pm 1.19$ & \\
\hline & $20-29$ & 13 & $2.62 \pm 0.68$ & & 86 & $3.00 \pm 0.94$ & \\
\hline & $30-39$ & 14 & $2.51 \pm 0.42$ & & 47 & $3.04 \pm 0.95$ & \\
\hline & $40-49$ & 17 & $2.54 \pm 0.80$ & & 41 & $3.18 \pm 1.21$ & \\
\hline & $50-59$ & 13 & $2.45 \pm 0.61$ & & 50 & $2.86 \pm 1.22$ & \\
\hline & $\geq 60$ & 24 & $2.31 \pm 0.77$ & & 29 & $3.16 \pm 1.32$ & \\
\hline
\end{tabular}

Values are presented as mean \pm standard deviation.

In cases of chronic inflammatory sinonasal diseases like AR or CRS with or without polyposis, nasal mucosal swelling occurs. This mucosal thickening can undergo hypertrophic change due to prolonged inflammatory insult [6]. The fibrotic change is recalcitrant to conventional treatment like nasal decongestants and steroids. Most of the time surgery is warranted to reverse this transformation [6]. When subjected to similar insult, NSB can undergo fibrotic changes as well, which would not only make this structure thicker but also less elastic in responding to nasal cycle. This may contribute to clinical nasal obstruction because of its proximity to the INV. Our study showed the widening of NSB in chronic diseases, which means narrowing of INV area.

A study in 2004, wherein CT scans of 595 subjects with CRS was reviewed for the prevalence of NSB. Among the 595 subjects, $332(55.79 \%)$ were considered to have NSB. The authors set a nonvalidated criterion where over $8 \mathrm{~mm}$ thickness of septum is determined as the presence of a swell body [7]. The authors of this study disagree with this statement because NSB is thought to be a part of the normal anatomy of the nasal septum, thus always present in healthy or diseased state. Another study performed in 2014 involving 40 patients with AR and 30 control subjects without AR, did not show any statistical significance between both groups (AR group, 11.23 \pm 1.56 ; control group, $11.17 \pm 1.49 ; P=0.869)$, which is in contrast to our study [8]. Small sample size may have underestimated the actual difference in both groups. As far as the authors are concerned, this is the only study available of NSB measurement in chronic sinonasal disease and control.

The majority of the previous studies on the morphology of NSB only consisted of radiographic measurement of the widest diameter. Our present study made some additional measurements that seemed to be relevant in describing the NSB in its fusiform structure. In this manner, we were able to determine that there are indeed significant changes in each segment/area measured in the NSB that were independent of each other. Also, in the present study, patients in the diseased group are in prolonged medication when CT scan was done. Therefore, any thickening observed could be due to mucosal remodeling due to chronicity of disease state.

When NSB measurement was analyzed by age group, the di- 

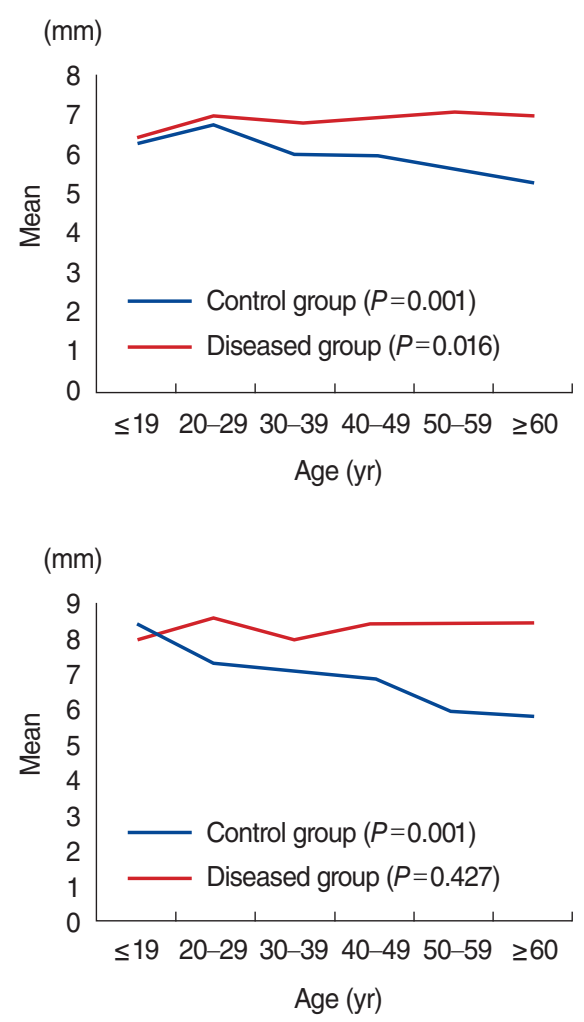

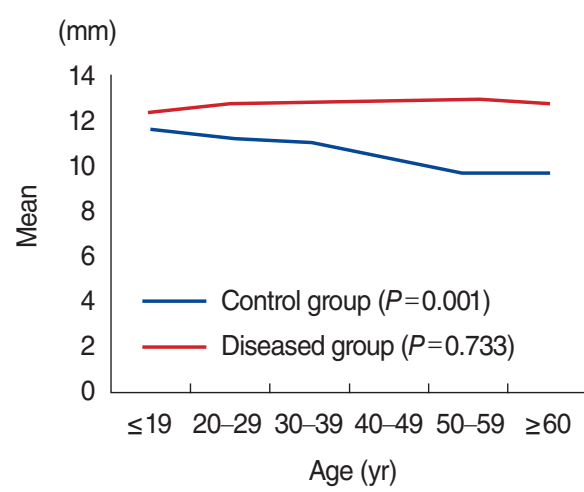

B

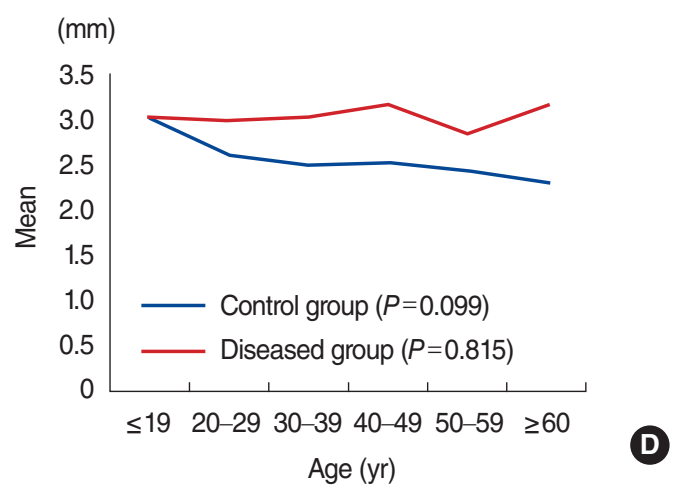

Fig. 2. Nasal septal body (NSB) diameter by age group between control and diseased group. (A) NSB anterior part, (B) NSB middle or widest part, (C) NSB posterior part, and (D) posterior part of septum.

ameter appears to diminish as the individual gets older in the control group. The result supports the widely known notion that mucosal epithelium atrophies with age [14]. However, the reverse trend was observed among those in the disease group which is in support to previous studies that mucosal remodeling such as basement membrane thickening, increased extracellular matrix deposition and goblet cell hyperplasia are noted to be more prominent in adult CRS compared to pediatric or adolescent CRS [15-17]. Thus, mucosal remodeling has a direct relationship with the duration of disease. The NSB diameter trend by age group among control and diseased group are shown in Table 2 and Fig. 2.

While the significance of NSB on nasal airflow remains debatable, the findings of this study suggest that it may be beneficial to take note of this structure in evaluating patients with nasal obstruction. Most of the times, clinicians would look for septal deviation, inferior turbinate hypertrophy and nasal masses in looking for the etiology of nasal obstruction. Even though this is the case, however, if none of the usual cases are present, clinicians sometimes mistake septal swell body as high deviation thus unnecessary septal surgery could probably be done.

Surgical treatment of NSB is not commonly done. This is due to lack of consensus among ENT clinicians or rhinologists where most consider surgical treatment of NSB as aggressive and controversial $[1,18]$. This lack of consensus may be influenced by in- consistent histological study results. The previous papers concluded that the primary role of NSB is humidification and regulation of the temperature of inspired air because the structure possesses more percentage of seromucinous glands than venous sinusoids $[2,4]$. They promote the preservation of these glands to prevent mucosal dryness and crust formation. On the other hand, other study showed high proportions of venous sinusoids within swell body thus highlights its capacity to alter nasal airflow [1]. The potential to affect nasal aerodynamics led some authors to address NSB surgically. To date, there are only 3 published articles that looked at the effect of NSB reduction. One report presented nasal cautery and cryotherapy of the NSB and inferior turbinate in the treatment of nasal obstruction and inflammatory disease showing no benefit [19]. However, this study is underpowered also involving multiple surgical techniques. Other study combined septal body volume reduction and turbinoplasty using microdebrider, which appears to be more efficient than turbinoplasty alone in patients with inferior turbinate and septal body hypertrophy [20]. Lastly, a study used radiofrequency ablation of septal swell body for correction of refractory nasal obstruction. They introduced an effective and safe in-office surgical treatment in reducing NSB [21]. Although, the results of these studies are promising, the long-term efficacies of these interventions have yet to be determined.

This study has limitation in some points. This study is basically 
radiologic evaluation and measuring area and distance is somewhat thin and short, which can cause a minor mistake of measurement. This study excluded PNS CT scans from patients whose septum cannot be delineated due to massive polyposis and severe septal deviation.

It is hard to draw definite conclusions about the clinical implication of NSB overdevelopment based on the results of the present study. However, the main focus of this study is to show that NSB overdevelopment could be observed in subjects with chronic sinonasal disease. The authors think that the findings of the current study provide additional information that could help further understanding of this mostly neglected structure.

\section{Conclusion and recommendation}

With aging, we expect nasal mucosal atrophy among normal subjects. However, patients with chronic sinonasal disease did show thickened nasal mucosa without any atrophy. Further study on the clinical impact of NSB among the elderly should be investigated. Likewise, a research design to define the role of septal body in nasal aerodynamics should be performed, and the possible treatment in cases of hypertrophy should be contemplated.

\section{CONFLICT OF INTEREST}

No potential conflict of interest relevant to this article was reported.

\section{ACKNOWLEDGMENTS}

The authors would like to acknowledge Rubiliza Onofre and Sohyeon Ahn for critical review and statistics contribution and Yun Jung Bae for her inputs about Seoul National University Bundang Hospital paranasal sinus computed tomography protocol.

\section{REFERENCES}

1. Costa DJ, Sanford T, Janney C, Cooper M, Sindwani R. Radiographic and anatomic characterization of the nasal septal swell body. Arch Otolaryngol Head Neck Surg. 2010 Nov;136(11):1107-10.

2. Wexler D, Braverman I, Amar M. Histology of the nasal septal swell body (septal turbinate). Otolaryngol Head Neck Surg. 2006 Apr;
134(4):596-600.

3. Setlur J, Goyal P. Relationship between septal body size and septal deviation. Am J Rhinol Allergy. 2011 Nov-Dec;25(6):397-400.

4. Elwany S, Salam SA, Soliman A, Medanni A, Talaat E. The septal body revisited. J Laryngol Otol. 2009 Mar;123(3):303-8.

5. Cole P. The four components of the nasal valve. Am J Rhinol. 2003 Mar-Apr;17(2):107-10.

6. Farmer SE, Eccles R. Chronic inferior turbinate enlargement and the implications for surgical intervention. Rhinology. 2006 Dec;44(4): 234-8.

7. Arslan M, Muderris T, Muderris S. Radiological study of the intumescentia septi nasi anterior. J Laryngol Otol. 2004 Mar;118(3):199-201.

8. San T, Gurkan E, Erdogan B, Tasel B. The effect of allergic rhinitis on nasal septal body size. J Med Updates. 2014;4(2):49-51.

9. Brozek JL, Bousquet J, Baena-Cagnani CE, Bonini S, Canonica GW, Casale TB, et al. Allergic Rhinitis and its Impact on Asthma (ARIA) guidelines: 2010 revision. J Allergy Clin Immunol. 2010 Sep;126(3): 466-76.

10. Kim DK, Rhee CS, Han DH,Won TB, Kim DY, Kim JW.Treatment of allergic rhinitis is associated with improved attention performance in children: the Allergic Rhinitis Cohort Study for Kids (ARCOKids). PLoS One. 2014 Oct;9(10):e109145.

11. Rosenfeld RM, Piccirillo JF, Chandrasekhar SS, Brook I, Ashok Kumar K, Kramper M, et al. Clinical practice guideline (update): adult sinusitis. Otolaryngol Head Neck Surg. 2015 Apr;152(2 Suppl):S1S39.

12. Ashraf N, Bhattacharyya N. Determination of the "incidental" Lund score for the staging of chronic rhinosinusitis. Otolaryngol Head Neck Surg. 2001 Nov;125(5):483-6.

13. Miman MC, Deliktas H, Ozturan O,Toplu Y,Akarcay M. Internal nasal valve: revisited with objective facts. Otolaryngol Head Neck Surg. 2006 Jan;134(1):41-7.

14. Pinto JM, Jeswani S. Rhinitis in the geriatric population. Allergy Asthma Clin Immunol. 2010 May;6(1):10.

15. Rehl RM, Balla AA, Cabay RJ, Hearp ML, Pytynia KB, Joe SA. Mucosal remodeling in chronic rhinosinusitis. Am J Rhinol. 2007 NovDec;21(6):651-7.

16. Chan KH, Abzug MJ, Coffinet L, Simoes EA, Cool C, Liu AH. Chronic rhinosinusitis in young children differs from adults: a histopathology study. J Pediatr. 2004 Feb;144(2):206-12.

17. Pawankar R, Nonaka M. Inflammatory mechanisms and remodeling in chronic rhinosinusitis and nasal polyps. Curr Allergy Asthma Rep. 2007 Jun;7(3):202-8.

18. Wotman M, Kacker A. Should otolaryngologists pay more attention to nasal swell bodies? Laryngoscope. 2015 Aug;125(8):1759-60.

19. Haight JS, Gardiner GW. Nasal cryosurgery and cautery: should the septum be treated and is a diagnosis relevant? J Otolaryngol. 1989 Jun;18(4):144-50.

20. Yu MS, Kim JY, Kim BH, Kang SH, Lim DJ. Feasibility of septal body volume reduction for patients with nasal obstruction. Laryngoscope. 2015 Jul;125(7):1523-8.

21. Catalano P, Ashmead MG, Carlson D. Radiofrequency ablation of septal swell body. Ann Otolaryngol Rhinol. 2015 Nov;2(11):1069. 